\title{
The Spectrum of Integrated Millimeter Flux of the Magellanic Clouds and 30-Doradus from TopHat and DIRBE Data
}

\author{
J. E. Aguirre, ${ }^{1}$ J. J. Bezaire, ${ }^{1}$ E. S. Cheng, ${ }^{2}$ D. A. Cottingham, ${ }^{3}$ S. S. Cordone, ${ }^{4}$ \\ T. M. Crawford, ${ }^{5}$ D. J. Fixsen, ${ }^{6}$ L. Knox, ${ }^{7}$ S. S. Meyer, ${ }^{1,5}$ H. U. Norgaard-Nielsen, ${ }^{8}$ \\ R. F. Silverberg, ${ }^{9}$ P. Timbie, ${ }^{4}$ G. W. Wilson ${ }^{10}$
}

\begin{abstract}
We present measurements of the integrated flux relative to the local background of the Large and Small Magellanic Clouds and the region 30-Doradus (the Tarantula Nebula) in the LMC in four frequency bands centered at 245, 400, 460, and $630 \mathrm{GHz}$, based on observations made with the TopHat telescope. We combine these observations with the corresponding measurements for the DIRBE bands 8,9 , and 10 to cover the frequency range $245-3000 \mathrm{GHz}(100-1220 \mu \mathrm{m})$ for these objects. We present spectra for all three objects and fit these spectra to a single-component greybody emission model and report best-fit dust temperatures, optical depths, and emissivity power-law indices, and we compare these results with other measurements in these regions and elsewhere. Using published dust grain opacities, we estimate the mass of the measured dust component in the three regions.
\end{abstract}

\footnotetext{
${ }^{1}$ University of Chicago, Department of Physics, 5640 South Ellis Avenue, Chicago, IL 60637

${ }^{2}$ Conceptual Analytics, LLC, 8209 Woburn Abbey Road, Glenn Dale, MD 20769

${ }^{3}$ Global Science and Technology, Inc., Laboratory for Astronomy and Solar Physics, NASA / Goddard Space Flight Center, Code 685, Greenbelt, MD 20771

${ }^{4}$ University of Wisconsin at Madison, Department of Physics, 1150 University Avenue, Madison, WI 53706

${ }^{5}$ University of Chicago, Department of Astronomy, 5640 South Ellis Avenue, Chicago, IL 60637

${ }^{6}$ SSAI, Laboratory for Astronomy and Solar Physics, NASA / Goddard Space Flight Center, Code 685, Greenbelt, MD 20771

${ }^{7}$ University of California, Department of Physics, One Shields Avenue, Davis, CA 95616

${ }^{8}$ Danish Space Research Institute, Juliane Maries Vej 32, DK-2100 Copenhagen, Denmark

${ }^{9}$ NASA / Goddard Space Flight Center, Laboratory for Astronomy and Solar Physics, Code 685, Greenbelt, MD 20771

${ }^{10}$ University of Massachusetts, Department of Astronomy, 619E LGRT-B, 710 North Pleasant Street, Amherst, MA 01003-9305
} 
Subject headings: Magellanic Clouds, dust extinction, ISM, infrared: galaxies, balloons

\section{Introduction}

There has been a recent upsurge in interest in interstellar dust emission, both in our own galaxy and in extragalactic environments. Galactic dust emission in the far-infrared (FIR) and microwave is a primary source of contamination to measurements of the Cosmic Microwave Background (CMB) (Masi et al. 2001; Jaffe et al. 2003). Association of the observed Cosmic Infrared Background (CIB) with dust emission from high-redshift galaxies (Puget et al. 1999; Scott et al. 2000) and the recognition of the potential use of this source as a probe of structure formation (Guiderdoni et al. 1998; Blain et al. 1999; Haiman \& Knox 2000; Knox et al. 2001) has placed a high priority on understanding extragalactic dust properties.

Most of what we know about interstellar dust from our own and other galaxies comes from measurements of extinction (absorption plus scattering) in the ultraviolet (UV), optical, and near- to mid-infrared (NIR, MIR) (Mathis 1990). Extinction measurements can be compared to dust models (Mathis et al. 1977; Draine \& Lee 1984; Li \& Draine 2001) to constrain the size, composition, and density of dust grains. Low-frequency $(\nu \ll 300 \mathrm{THz})$ dust emission is thought to be dominated by thermal greybody emission from dust grains heated by the interstellar radiation field (IRF), so to turn the knowledge of the dust from extinction into a prediction for low-frequency dust emission, one needs two further pieces of information: the spectrum of the IRF and the greybody emissivity of the different grain populations - in general a function of frequency, grain size, and grain composition. Conversely, one can use measurements of low-frequency dust emission combined with models of the dust density and optical properties to constrain models of the IRF.

In the simplest models of optical properties of dust at low frequencies, the dust emissivity is independent of grain size and composition. Hildebrand (1983) and Draine \& Lee (1984) (hereafter DL84) argue that for dust grains much smaller than a wavelength $(\lambda=c / \nu \gg a$, where $a$ is the grain radius), the emission cross-section will be proportional to the volume of the grain. The emissivity per unit dust mass for a given dust density is then independent

of the nature of the grain size distribution as long as all grains are small compared to the wavelengths of interest. DL84 argue further that electric dipole radiation will dominate in this limit, and the dust emissivity will be proportional to the square of the frequency, regardless of dust composition. Later theoretical work (Tielens \& Allamandola 1987) and laboratory measurements (Agladze et al. 1996) have suggested different power-law indices for 
IR dust emissivity, depending on composition, and ranging from $\nu^{1}$ to $\nu^{2.7}$, even in the regime $\lambda=c / \nu \gg a$. Agladze et al. (1996) have also found evidence for temperature-dependent behavior of the power-law index for certain types of amorphous silicate grains.

Further complicating matters is the possibility that different dust populations along a single line of sight can have different emission temperatures, even if the radiation field does not change along the line of sight. Grains in the diffuse interstellar medium (ISM) are predicted to have temperatures of $\sim 10-20 \mathrm{~K}$ (DL84), but very small grains can be transiently heated to temperatures of hundreds of Kelvin by a single UV photon and will cool to ambient temperature by re-emitting in the NIR/MIR (Sellgren 1984). Li \& Draine (2001) estimate the maximum grain radius $a$ at which this effect is noticeable to be $a \sim 25 \mathrm{~nm}$. They divide silicate and carbonaceous (graphite) dust into "big" and "small" populations based on this criterion and estimate emission from small grains to be an important contributor at $5000 \mathrm{GHz}$ but small compared to large-grain emission at $3000 \mathrm{GHz}$ and lower frequencies.

Dunne \& Eales (2001) have combined data from the Infrared Astronomy Satellite (IRAS) at $5000 \mathrm{GHz}(60 \mu \mathrm{m})$ and $3000 \mathrm{GHz}(100 \mu \mathrm{m})$ with $670 \mathrm{GHz}(450 \mu \mathrm{m})$ and $350 \mathrm{GHz}(850 \mu \mathrm{m})$ data from the Sub-millimetre Common User Bolometer Array (SCUBA) camera and other sub-mm measurements in the literature to characterize the dust emission from 32 nearby galaxies. In an earlier study, Dunne et al. (2000) found that fitting just the two IRAS points and the SCUBA $350 \mathrm{GHz}$ point resulted in derived temperatures of $35.6 \pm 4.9 \mathrm{~K}$ and emissivity indices of $1.3 \pm 0.2$ for a larger sample of 104 nearby objects. The new study finds that for the sources for which they were able to add more sub-mm points, the spectra were better fit by a model with two components at different temperatures, with $31 \mathrm{~K}<T_{\text {hot }}<60 \mathrm{~K}$ and $18 \mathrm{~K}<T_{\text {cold }}<32 \mathrm{~K}$. The authors note that these conclusions are not definitive, due both to a paucity of measurements for most of their sources (only 10 of their sources are measured in more than four spectral bands - only one in more than six bands - and a two-component greybody model with power-law emissivity in general has six free parameters) and to a fundamental degeneracy between a broadened temperature distribution and a shallower emissivity power-law.

In this paper we consider high-fidelity measurements in seven frequency bands of the two galaxies nearest to ours: the Large and Small Magellanic Clouds. All of the measurements are at frequencies $\nu \leq 3000 \mathrm{GHz}$. The measurements at $\nu \geq 1250 \mathrm{GHz}$ are taken from the publicly available data from the Diffuse Infrared Background Experiment (DIRBE) on the Cosmic Background Explorer (COBE) satellite, while the measurements at $\nu<1250 \mathrm{GHz}$ are new results from the TopHat instrument.

The irregular dwarf galaxies known as the Magellanic Clouds are the most prominent extragalactic features in the southern sky and have been observed for hundreds of years 
in many spectral bands (for a summary, see Westerlund (1997) or van den Bergh (2000)). Despite the proximity of these two galaxies, there exist relatively few observations of them in the FIR or in continuum sub-millimeter or microwave bands.

Studies of dust in the Magellanic Clouds have been done using extinction measurements (Rodrigues et al. 1997; Gordon \& Clayton 1998; Misselt et al. 1999; Weingartner \& Draine 2001). Weingartner \& Draine (2001) estimate dust grain size distributions in the Magellanic Clouds and our galaxy and find that the distribution shape and large-radius cutoff are similar in the two environments. The product of grain size distribution and emissivity per grain increases with grain size in the two types of grains in the Weingartner \& Draine model (silicates and carbonaceous (graphite) grains) up to a cutoff at $a \sim 0.2 \mu \mathrm{m}$ for silicates and $a \sim 1 \mu \mathrm{m}$ for graphites. Based on this work, we assume grains large enough to be in thermal equilibrium with the IRF will dominate the emission at $\nu \leq 3000 \mathrm{GHz}$ from the Magellanic Clouds.

Attempts have been made to characterize the dust in the Magellanic Clouds using IRAS data alone, (Sauvage et al. 1990; Stanimirovic et al. 2000), while Andreani et al. (1990) combined IRAS data with single scans across the LMC and SMC with a groundbased millimeter-wave receiver operating from Antarctica. IRAS observed the entire sky at $5^{\prime}$ resolution in four spectral bands centered at 25000, 12000, 5000, and $3000 \mathrm{GHz}(12,25,60$, and $100 \mu \mathrm{m})$. However, IRAS was designed to detect point sources, and though large-area sky maps have been created from IRAS data (ISSA images, Wheelock et al. (1994)), large zero-point and calibration drifts across these maps render them unsuitable for determinations of absolute flux in regions as large as the Magellanic Clouds.

DIRBE extended the frequency range of IRAS with two lower-frequency channels (2140 $\mathrm{GHz}(140 \mu \mathrm{m})$ and $1250 \mathrm{GHz}(240 \mu \mathrm{m}))$ and was specifically designed to measure diffuse emission, so the zero-point and absolute calibration are more well-behaved and well-characterized. The angular resolution of DIRBE is $0.7^{\circ}$, which is well-matched to characterizing the integrated dust properties of the Magellanic Clouds, which have angular extents on the order of degrees. Schlegel et al. (1998) (hereafter SFD98) have combined IRAS and DIRBE $3000 \mathrm{GHz}$ $(100 \mu \mathrm{m})$ data to produce a full-sky model with $5^{\prime}$ resolution and the stability of the DIRBE data. Finkbeiner et al. (1999) (hereafter FDS99) use DIRBE $1250 \mathrm{GHz}$ data and data from the COBE Far Infrared Absolute Spectrophotometer (FIRAS) to extend this model to longer frequencies, but they exclude the Magellanic Clouds from the extended model. Stanimirovic et al. (2000) and Li \& Draine (2002) use DIRBE data and the SFD98 model to characterize the dust in the SMC, and Stanimirovic et al. (2000) find that some combination of components at temperatures between $15 \mathrm{~K}$ and $30 \mathrm{~K}$ and an emissivity proportional to $\nu^{2}$ fits the combined IRAS/DIRBE/SFD98 data at $\nu \leq 5000 \mathrm{GHz}$. Similar analyses have not been 
published for the integrated dust emission from the LMC.

We report here a measurement of the spectra of integrated flux relative to the background of the LMC and SMC. We report separate results for the LMC with the active starforming region 30-Doradus (the Tarantula Nebula) masked off and for 30-Doradus alone. This measurement is based on observations made with TopHat and DIRBE, and spans the range $245-3000 \mathrm{GHz}$. We report calibrated spectra (in Jy, $1 \mathrm{Jy}=10^{-26} \mathrm{Wm}^{-2} \mathrm{~Hz}^{-1}$ ) of all three regions. We fit the calibrated spectra to a single-component greybody emission model with power-law emissivity and report a best-fit optical depth, temperature, and emissivity power-law index for each region. These new measurements provide data which span a gap in our knowledge of the LMC and SMC integrated spectra. The dust emission properties are complicated by neither stochastic, non-equilibrium dust grain heating nor resonance emission, which are important factors at frequencies above $3 \mathrm{THz}$. In addition, the sub-mm measurements in the Rayleigh-Jeans portion of the dust emission permit an estimation of the dust mass. In particular, if a steep emission spectrum is indicated for theoretical reasons, a large mass of cold gas would be required to match the flat sub-mm spectrum reported here.

The paper is arranged as follows. Section 2 describes the TopHat instrument and observations and Section 3 describes the reduction of raw TopHat data to uncalibrated sky maps and uncertainties. Section 4 describes the flux analysis performed and the regions selected. Section 5 explains how we treat the DIRBE data and combine it with the TopHat observations. The TopHat calibration and the effect of the DIRBE calibration uncertainties are discussed in Section 6, and the final calibrated fluxes with errors are given in Section 7. We discuss the physical interpretation of these results and compare to results from the literature in Section 8, and we discuss applications of these results and future work in Section 9.

\section{Instrument Description and Observations}

TopHat (Cheng 1994; Silverberg et al. 2003) is a balloon-borne telescope designed to measure millimeter astrophysical emission over a large area of sky. TopHat is an on-axis Cassegrain telescope with a $1 \mathrm{~m}$ aluminum primary and a secondary mirror suspended on six Kevlar fibers. The beam was designed to be an approximately $20^{\prime}$ FWHM top hat, and ground measurements of the beam profile were consistent with the predicted shape. In addition, ground measurements were performed of the far sidelobe response, and the rejection was measured to be $>80 \mathrm{~dB}$ at angles greater than $25^{\circ}$ from the optical axis, and $>110 \mathrm{~dB}$ at angles greater than $70^{\circ}$. The instrument is mounted on top of a scientific balloon, with a gondola providing support electronics, power, and telemetry hanging beneath. The telescope and a conical radiation shield are mounted on a rotating azimuthal mount, with the optical 
axis fixed at a $12^{\circ}$ inclination from the rotation axis. Observations are made by rotating the mount at a constant rate of one rotation per $16 \mathrm{sec}$. Observing at $78^{\circ} \mathrm{S}$ latitude, the scan pattern as the earth rotates becomes a series of interlocked circles whose centers circumscribe the South Celestial Pole (SCP) once each sidereal day. The nominal observed region is then approximately a $48^{\circ}$ diameter circle, centered on the SCP. The actual scan pattern obtained was more complicated and covered a slightly larger area due to a tilt of the telescope from horizontal and the rotation of the balloon.

The instrument observes a single pixel in five spectral bands, each with a single detector. The band is defined by an IR absorber, resonant grid beam splitters, and band defining filters. In this work, we define the center frequency of the band $\langle\nu\rangle$ as an effective Rayleigh-Jeans (RJ) frequency by

$$
\langle\nu\rangle=\left[\frac{\int \nu^{2} t(\nu) d \nu}{\int t(\nu) d \nu}\right]^{1 / 2}
$$

where $t(\nu)$ is the transmission of the band. The center frequencies are then 175, 245, 400, 460 , and $630 \mathrm{GHz}$, with $\Delta \nu / \nu \sim 25 \%$ in the two lowest-frequency channels and $\sim 10 \%$ in the highest three. The spectral bandpasses were determined before the flight using Fourier transform spectrometry; the bandpasses of the four highest-frequency bands, which are used in this analysis, are shown in Figure 1. The width of the bands leads to appreciable color corrections for source spectra that differ appreciably from RJ. These corrections are incorporated into spectral fits as described in Section 6; the calculation of the corrections themselves (for both TopHat and DIRBE) is discussed in Appendix A. The detectors are five silicon bolometers with ion-implanted thermistors cryogenically cooled to $270 \mathrm{mK}$ by a ${ }^{3} \mathrm{He}$ cryostat; the dewar and its internal electronics are described in detail in Fixsen et al. (2001) and Oh et al. (2001). The band at $175 \mathrm{GHz}$ is not used in this analysis because of excess noise. 


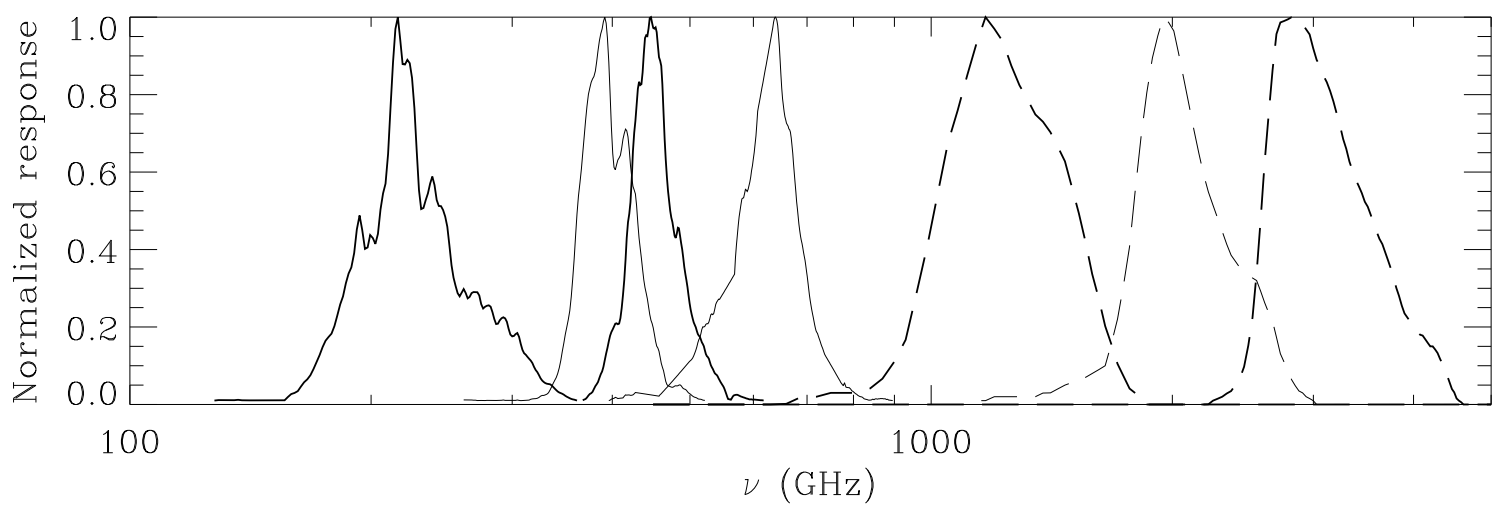

Fig. 1. - Bandpass relative to a white spectrum of the four TopHat bands used in this analysis, together with DIRBE bands 8, 9, and 10. The TopHat bands are shown in alternating heavy and light solid lines, and the DIRBE bands in heavy and light dashed lines. The transmission of each band has been normalized to unity at the peak. 
The telescope is not actively pointed. The location of the beam is reconstructed after the flight using a two-axis tilt meter, GPS information, and the location of the sun as determined by four small imaging telescopes on the radiation shield of the telescope, which record the sun position four times each rotation. A pointing model was constructed which uses the data from these sensors and depends on a small number of parameters to locate the spin axis and the beam. The parameters are determined by a multi-dimensional fit of the observations containing the Galaxy to a map. The observations of the Magellanic Clouds are not used in the pointing fit; rather, we take the reproducibility of their location on repeated observations as independent evidence of the quality of the pointing model. The RMS pointing errors are determined to be $\sim 3-4^{\prime}$, much smaller than the beam, and are negligible for this analysis.

TopHat was launched from McMurdo Station, Antarctica at 06:55 UT 2001 January 4 by the National Scientific Balloon Facility (NSBF). After an initial checkout, sky observations began at 14:10 UT 2001 January 4 and continued until 14:00 UT 2001 January 8 when the cryogens were exhausted. All observations occurred at a float altitude of $37.5 \pm 1.4 \mathrm{~km}$. The telescope was turned off on 2001 January 10, and the balloon cut down and the flight disks recovered on 2001 January 31. The observations consist of a nearly circular patch of sky centered at the South Celestial Pole (SCP), approximately $30^{\circ}$ in radius, or about $6 \%$ of the sky, re-observed four times over the course of $\sim 4$ sidereal days. Because of interruptions in the observations for system checks, the data exists in only three continuous sections. In addition, data taken during the first 16 hours of observation proved unusable due to longsettling transients in many of the systems. We have divided the remaining observations into two independent sets, denoted Epoch I and Epoch II, with approximately equal observing weight, so that all steps in the analysis may be performed on both epochs and the results compared. These divisions are summarized in Table 1. 
Table 1. Observing times of data used in this analysis.

\begin{tabular}{ccc}
\hline \hline Epoch & Start & End \\
\hline \multirow{2}{*}{ I } & 04:18 UT 2001 Jan 5 & 15:28 UT 2001 Jan 5 \\
& 15:43 UT 2001 Jan 5 & 00:33 UT 2001 Jan 6 \\
& 17:55 UT 2001 Jan 7 & 14:00 UT 2001 Jan 8 \\
\hline II & 00:33 UT 2001 Jan 6 & 17:28 UT 2001 Jan 6 \\
& 18:03 UT 2001 Jan 6 & 17:55 UT 2001 Jan 7 \\
\hline
\end{tabular}




\section{Data Analysis}

The raw radiometer data are reduced by constructing a minimum variance fit of the timestream to a sky map. Prior to mapmaking, the data are processed as follows. Cosmic ray strikes and other anomalies are removed from the time stream data and the instrument transfer function is deconvolved. Then instrumental effects in the data are identified; large instrumental signals of various kinds which are localized in time are excised and ignored in further analysis, while scan-synchronous instrumental signal is dealt with by fitting the timestream simultaneously to the sky and a model of this scan-synchronous signal. and contaminated data either cut or an appropriate model constructed by which the contaminant may be removed in a simultaneous fit.

The timestream noise is estimated iteratively from a series of intermediate fit residuals. A model of the telescope pointing is constructed and its parameters fit to minimize the map errors. The final sky map is a vector $m$, produced by a linear fit to the accepted data in the cleaned timestream $d$ which minimizes the $\chi^{2}$ function

$$
\chi^{2}=(d-A(p) m)^{T} W(d-A(p) m)
$$

where $d$ is the cleaned data timestream, $A$ is the pointing matrix, a function of the pointing parameters $p$, which is supplemented by model templates to account for instrumental effects, and $W$ is the inverse of the time-time noise covariance matrix, $W=\left\langle n n^{T}\right\rangle^{-1}$. The pointing matrix is pixelized using HEALPix ${ }^{11}$ (Górski, Hivon, \& Wandelt 1999) with pixels $14^{\prime}$ on a side. The best fit map is given by

$$
m=\left(A^{T} W A\right)^{-1} A^{T} W d=N A^{T} W d
$$

where the covariance matrix $N$ for this simultaneous estimation of sky pixels plus instrumental model parameters is given by

$$
N=\left(A^{T} W A\right)^{-1}
$$

Equations 3 and 4 are solved directly using MADCAP (Borrill 1999). Maps are produced for both Epochs separately. In the following, we marginalize $N$ over sky pixels which do not lie in the fields of interest and over all instrumental parameters.

\footnotetext{
${ }^{11}$ http: //www.eso.org/science/healpix/
} 


\section{Flux Analysis}

TopHat has no absolute reference for the power coming from the sky; it is sensitive only to flux differences between different positions. Our formal uncertainty on any quantity with a nonzero projection onto a constant sky flux is infinite; therefore we must construct a purely differential quantity to estimate. The integrated flux from an extended source minus the integrated flux from a surrounding set of pixels with the same total solid angle as the source is such a quantity. This approach is particularly appropriate in the case that the flux from the on-source pixels is a combination of the source emission and emission from a relatively uniform, optically thin foreground; then the differential measurement gives the flux from the source only.

There is some evidence of systematic contamination above the random noise level in the TopHat data, particularly in spatial modes which are a function of declination (Dec) only. A large spin synchronous instrument signal is fit out of each channel's timestream simultaneously with the best-fit map. While the pixel-pixel correlations induced by this instrument model are included in the pixel covariance matrices used in the flux analysis, the model lacks sufficient fidelity to remove the spin-synchronous signals completely, leaving a small residual spin synchronous signal improperly subtracted and unaccounted for in the covariance matrix. The symmetry of our scanning strategy naturally projects any residual spin synchronous signal mainly onto spatial modes in the map that are functions of declination only. For this reason, we have constructed the on- and off-source regions to have the same number of pixels on each iso-latitude ring in the HEALPix "Ring" pixelization, rendering the difference between on- and off-source flux insensitive to these potentially contaminated modes. We arrange the off-source pixels symmetrically around the on-source pixels so that the differential flux is also insensitive (to first order) to other long-wavelength modes that we do not wish to include in this measurement, such as the CMB dipole. The diameters of the on-source regions (chosen to be circular for convenience) were optimized for the "target" Magellanic Cloud regions by varying the diameter around an initial value selected by eye to enclose the particular high-contrast area of the DIRBE $1250 \mathrm{GHz}$ map and noting when the slope of the enclosed flux versus diameter tends to zero. This process is not used to optimize the 30-Doradus on-source region because it is itself embedded in a high-contrast region. The diameter of the 30-Doradus on-source region is chosen by eye.

Having chosen the source region and a background region satisfying the above conditions, we then extract from the full map $m$ and covariance matrix $N$ (Equations 3 and 4 ) the pixels corresponding to the on and off-source regions. This is done separately for the two Epochs. We denote the vector of extracted pixel values $v$, and the matrix of extracted pixel-pixel covariances $C$. The weighting vector $w$ is defined such that it is 1 for the on-source pixels 
and -1 for the off-source pixels. We then calculate, for each channel, the quantity

$$
S=w^{T} v
$$

with a corresponding error variance of

$$
\sigma_{S}^{2}=w^{T} C w
$$

We demonstrate the insensitivity of the weighting vector $w$ for the LMC, SMC, and 30Doradus regions to low spatial frequency modes by giving the normalized projections $\left(w^{T} \xi\right) / \sqrt{\left(w^{T} w\right)\left(\xi^{T} \xi\right)}$ of various modes $\xi$ onto the weight vector $w$ in Table 2. 
Table 2. Normalized projections of low spatial frequency modes onto the pixel weight vectors used in the differential flux measurements.

\begin{tabular}{lrrrr}
\hline \hline \multicolumn{1}{c}{ Mode } & LMC & 30-Dor & SMC & Blank \\
\hline Constant offset & 0 & 0 & 0 & 0 \\
CMB dipole & -0.0093 & 0.0017 & 0.0147 & 0.0071 \\
Cross dipole 1 & 0.0136 & 0.0012 & 0.0079 & 0.0126 \\
Cross dipole 2 & 0.0002 & $<1 \times 10^{-4}$ & 0.0027 & 0.0003 \\
Constant dec mode & 0 & 0 & 0 & 0 \\
\hline
\end{tabular}

Note. - The "cross dipole" modes are the two dipole directions orthogonal to the $\mathrm{CMB}$ dipole. Cross dipole 2 points primarily along the direction towards the SCP. 
There are no sufficiently bright point sources between 245 and $630 \mathrm{GHz}$ in the TopHat observing region, so we constrain the possible in-flight beam profile by a combination of ground measurements and limits on how the optical configuration could possibly change during launch and at altitude. However, in this analysis we have chosen the conservative approach of making all source regions large compared to an upper limit on the beam size determined by assuming the beam is the size of the most compact bright sources we can find in our maps. These most compact sources are no more than one degree FWHM, and all source regions in this analysis are at least two degrees in diameter. Because of the very high sidelobe rejection of the telescope, the contribution of the brightest off-axis sources (the sun and the Galaxy) are negligible.

In addition to the source regions of the LMC, SMC, and 30-Doradus, we have selected five regions of appreciable Galactic dust emission which are out of the Galactic plane and one "blank" region on which to perform the flux analysis. The Galactic dust regions are used in the calibration, discussed in Section 6. These regions are numbered 1-5, with regions 3, 4 , and 5 corresponding to the Chameleon Nebulae. The blank region is chosen such that its differential brightness is consistent with zero in the DIRBE $1250 \mathrm{GHz}$ channel. The TopHat flux of the blank region serves as a consistency check on the method, since we expect to be dominated by thermal dust emission correlated with DIRBE in the TopHat bands.

The on-source fields are all circular about the center given; their location and the solid angle they subtend are given in Table 3. The on- and off-source fields for each region are shown in Figure 2. 
Table 3. Locations of regions used in the flux analysis.

\begin{tabular}{lccccccc}
\hline \hline Region & \multicolumn{3}{c}{ RA Center } & \multicolumn{3}{c}{ Dec Center } & \multirow{2}{*}{$\begin{array}{c}\text { Solid angle } \\
(\text { ster })\end{array}$} \\
& $(\mathrm{h})$ & $(\mathrm{min})$ & $(\mathrm{s})$ & $(\mathrm{deg})$ & $(\mathrm{min})$ & $(\mathrm{s})$ & ( \\
\hline Dust 1 & 7 & 7 & 52.1 & -78 & 50 & 6.4 & .01596 \\
Dust 2 & 19 & 30 & 0.0 & -80 & 7 & 11.0 & .01596 \\
Dust 3 & 11 & 4 & 24.7 & -77 & 32 & 56.8 & .00118 \\
Dust 4 & 12 & 49 & 5.5 & -79 & 56 & 10.6 & .00113 \\
Dust 5 & 12 & 54 & 0.0 & -77 & 10 & 53.0 & .00118 \\
Blank & 23 & 16 & 24.4 & -66 & 26 & 36.7 & .00644 \\
\hline LMC & 5 & 18 & 27.7 & -68 & 29 & 35.6 & .01481 \\
SMC & 0 & 52 & 15.5 & -72 & 56 & 31.6 & .00544 \\
30-Dor & 5 & 39 & 28.4 & -69 & 3 & 3.5 & .00120 \\
\hline
\end{tabular}

Note. - All coordinates are J2000. 


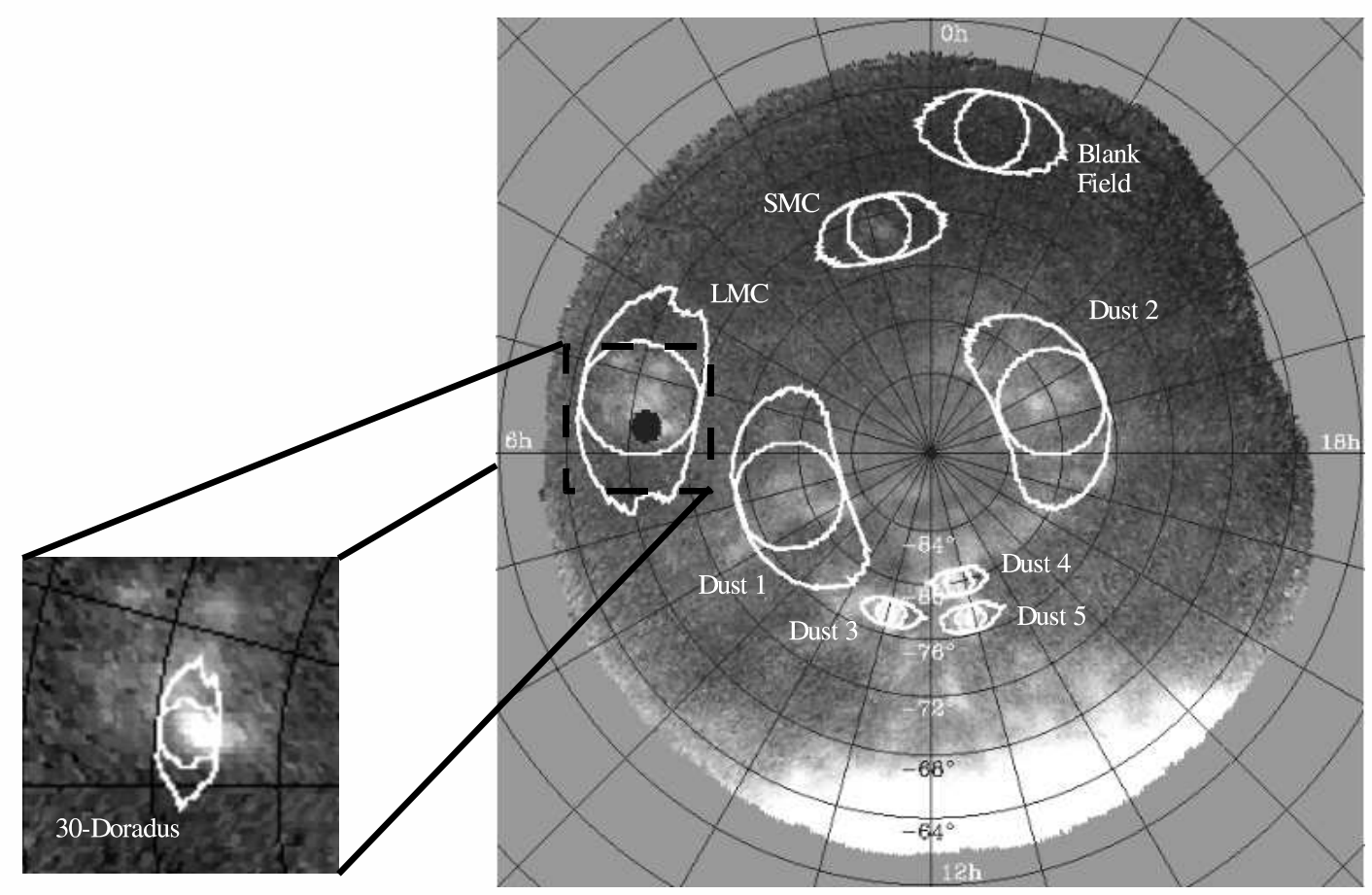

Fig. 2.- TopHat channel 5 combined Epoch I and II map showing the on-source and offsource regions used in calculating the LMC, SMC, and blank field flux, as well as the dust regions used for calibration. The region used for 30-Doradus is shown in the inset. RA increases counter-clockwise from the top in steps of $1 \mathrm{~h}$ per division; the SCP is at the center of the map, with Dec increasing by $4^{\circ}$ per division. In computing the flux of the LMC alone, the 30-Doradus region (the central circle in the inset) is not included. 
We probe the consistency of the flux results obtained by several $\chi^{2}$ tests. We first ask, for a given Epoch, if the spectrum of the blank field in all four TopHat channels is consistent with zero flux. The results of these tests are presented in Table 4, which gives the $\chi^{2} /$ d.o.f and the probability to exceed (PTE) this value with a correct model. We find that the combined TopHat measurements of the blank region are consistent with zero in both Epochs, but we note that this is not the case for an arbitrary choice of off-source region shape, such as a region circularly symmetric about the on-source pixels. This choice of offsource region yields a $\chi^{2} /$ d.o.f $=18.7 / 4$ for the model that the blank region is indeed blank in Epoch II, with an associated PTE of 0.001. We next ask if the flux measurements are consistent between Epochs. These results for all regions selected are given in Table 5. We find that the spectra measured are consistent between Epochs and therefore in what follows we quote the fluxes from a weighted average of the two Epochs. Again, this conclusion is not reached with off-source regions circularly symmetric about the on-source region. For example, the LMC minus 30-Doradus has an Epoch-to-Epoch consistency $\chi^{2} /$ d.o.f $=19.7 / 4$ with this naive choice of off-source regions, with an associated PTE of $5 \times 10^{-4}$. Finally, we note that, as measured in the three DIRBE bands, the choice of off-source region does not affect the measured differential flux above the $\sim 1 \%$ level, except for the special case of 30-Doradus, for which the different off-source regions sample different parts of the still reasonably bright diffuse LMC. 
Table 4. $\chi^{2}$ test of the null hypothesis for the blank field.

\begin{tabular}{lcc}
\hline \hline Epoch & $\chi^{2} /$ d.o.f & PTE \\
\hline I & $5.165 / 4$ & 0.27 \\
II & $1.438 / 4$ & 0.84 \\
Sum & $6.603 / 8$ & 0.58 \\
\hline
\end{tabular}

Table 5. Results of $\chi^{2}$ tests of consistency between TopHat Epochs for observed fields.

\begin{tabular}{lcc}
\hline \hline Region & $\chi^{2} /$ d.o.f & PTE \\
\hline Dust 1 & $8.556 / 4$ & 0.07 \\
Dust 2 & $4.155 / 4$ & 0.39 \\
Dust 3 & $4.803 / 4$ & 0.31 \\
Dust 4 & $1.051 / 4$ & 0.90 \\
Dust 5 & $4.344 / 4$ & 0.36 \\
Blank & $4.699 / 4$ & 0.32 \\
\hline LMC & $3.757 / 4$ & 0.44 \\
SMC & $3.202 / 4$ & 0.52 \\
30-Dor & $0.901 / 4$ & 0.92 \\
\hline
\end{tabular}




\section{Treatment of the DIRBE Data}

Having demonstrated the internal consistency of the TopHat data, we proceed to combine it with the DIRBE data set to extend the range of the spectrum. The DIRBE observations are the closest in frequency to our own, extending up in frequency from $1250 \mathrm{GHz}$, and with a beam of $0.7^{\circ}$ they are well-suited to measurements of objects on the angular scales of the Magellanic Clouds (Kelsall et al. 1998; Hauser et al. 1998). We use the Zodi-Subtracted Mission Average (ZSMA) intensity and standard deviation data from bands 8, 9, and 10, with nominal band centers of 1250, 2140, and $3000 \mathrm{GHz}$. The bandpasses are shown in Figure 1. DIRBE reports their nominal band centers relative to source spectrum with constant $\nu I_{\nu}$; the color corrections for a finite set of other source spectra are available as part of the DIRBE Explanatory Supplement (Hauser et al. 1998a) or one can calculate corrections for an arbitrary spectrum from the published DIRBE passbands, available electronically. ${ }^{12}$ We incorporate corrections for various model spectra in a spectral fit as described in Section 6; the calculation of the corrections is discussed in Appendix A.

The DIRBE maps exist in the COBE quadcube pixelization, so for the purposes of comparison it must be re-pixelized. We re-pixelize by resampling the quadcube pixelization onto the $14^{\prime}$ HEALPix pixelization; the standard deviations per pixel are similarly repixelized. We note that the $14^{\prime}$ pixels used oversample the DIRBE beam. We then select the same regions as for the TopHat analysis and perform the differencing of the integrated flux in an identical fashion. This allows us to combine the DIRBE measurements straightforwardly with our own. We note that for the differential analysis we make, the errors in flux due to the DIRBE absolute offset uncertainty and to zodiacal subtraction uncertainty are negligible.

The gain calibration errors, however, are not negligible and must be considered in addition to the random errors given by the standard deviation on the ZSMA maps. Our estimate of the DIRBE gain calibration error is based on the work of Hauser et al. (1998) and the cross-calibration of the DIRBE gains using FIRAS (Fixsen et al. 1997). The cross-calibration with FIRAS at 2140 and $1250 \mathrm{GHz}$ resulted in slightly different best-fit DIRBE gains and improved error bars on those gains. The Hauser et al. (1998) error at $3000 \mathrm{GHz}$ was not improved because half of the DIRBE passband was outside the FIRAS spectral coverage. The gains and errors found by the various authors are given in Table 6 . We describe how these gains and uncertainties are incorporated into the analysis in Section 6.

\footnotetext{
${ }^{12}$ http://space.gsfc.nasa.gov/astro/cobe/dirbe_exsup.html
} 
Table 6. DIRBE gains and uncertainties.

\begin{tabular}{|c|c|c|c|c|c|c|}
\hline \multirow[t]{2}{*}{ Reference } & \multicolumn{2}{|c|}{$3000 \mathrm{GHz}$} & \multicolumn{2}{|c|}{$2140 \mathrm{GHz}$} & \multicolumn{2}{|c|}{$1250 \mathrm{GHz}$} \\
\hline & Gain & Uncertainty & Gain & Uncertainty & Gain & Uncertainty \\
\hline Hauser et al. 1998 & 1.00 & 0.135 & 1.00 & 0.106 & 1.00 & 0.116 \\
\hline Fixsen et al. 1997 & 1.25 & 0.150 & 1.04 & 0.02 & 1.06 & 0.02 \\
\hline This work, as prior & 1.00 & 0.135 & 1.04 & 0.02 & 1.06 & 0.02 \\
\hline This work, best fit & 1.21 & $\begin{array}{l}+.01 \\
+.09\end{array}$ & 1.03 & $\begin{array}{l}+.02 \\
-.00\end{array}$ & 1.05 & $\begin{array}{l}+.02 \\
-.00\end{array}$ \\
\hline
\end{tabular}

Note. - All gains and uncertainties given are dimensionless and are referred to the Hauser et al. 1998 values. 


\section{Calibration}

In the $245 \mathrm{GHz}$ channel, the CMB dipole is observed with high signal-to-noise and is the dominant feature in that channel's map, apart from the Galaxy, so it is used as a calibration source. The $245 \mathrm{GHz}$ calibration uses the best measurement of COBE (Fixsen \& Mather 2002) for the amplitude and direction of the dipole and applies a correction due to the earth's rotation around the sun. The error on the calibration of the $245 \mathrm{GHz}$ channel is appreciably smaller than the random errors in the flux measurements of this channel, so we ignore this error in the following analysis.

In the higher-frequency channels, the CMB dipole is not detected with significance, so another calibration must be used. This is complicated by the absence of known calibration sources in the range $400-630 \mathrm{GHz}$ at angular scales of $1^{\circ}$. We therefore use an interpolation between the Galactic dust measurements in the calibrated $245 \mathrm{GHz}$ channel and the calibrated DIRBE channels at 1250, 2140, and $3000 \mathrm{GHz}$. This calibration uses the fields labelled Dust 1 - 5 in Table 3 and Figure 2, and does not use the measurements of the "target" Magellanic Cloud regions.

The spectral interpolation requires a model of this Galactic dust. We assume each of the dust regions is optically thin at all frequencies of interest and has uniform dust temperature and optical properties throughout, and we model the integrated flux from each region as

$$
\begin{aligned}
F_{\nu} & =\int d \Omega d \ell \rho_{d}(\Omega, \ell) \kappa_{m} B_{\nu}(T) \\
& =\Delta \Omega \tau_{\nu} B_{\nu}(T)
\end{aligned}
$$

where $\tau_{\nu}$ is the mean optical depth along all lines of sight through the source at frequency $\nu, B_{\nu}(T)$ is the Planck blackbody brightness at frequency $\nu$ and temperature $T, \kappa_{m}$ is the dust opacity in $\mathrm{cm}^{2} / \mathrm{g}, \rho_{d}$ is the dust mass density, and the integral is taken over lines of sight and solid angle. We assume a power-law emissivity to the dust so that

$$
\begin{aligned}
\tau_{\nu} & =\int d \ell \rho_{d}(\ell) \kappa_{m}(\nu) \\
& =\int d \ell \rho_{d}(\ell) \kappa_{m}\left(\nu_{0}\right)\left(\nu / \nu_{0}\right)^{\alpha} \\
& =\tau\left(\nu_{0}\right)\left(\nu / \nu_{0}\right)^{\alpha}
\end{aligned}
$$

where $\nu_{0}=600 \mathrm{GHz}$.

We then fit the measured flux from the five dust regions to the dust model in Equation 7 , where each region is fit to its own spectrum with free parameters $T, \alpha$, and $\tau\left(\nu_{0}\right)$, and

each of the three unknown TopHat calibrations and the three DIRBE calibrations is allowed 
to vary but forced to be identical for the five regions. We assume a Gaussian prior on the DIRBE calibrations of the best combination of the Hauser et al. (1998) and Fixsen et al. (1997) values, as given in Table 6. This leads to minimizing the $\chi^{2}$ function

$$
\chi^{2}=\sum_{i=1}^{5} \sum_{j=1}^{7}\left(\frac{F(i, j) / K(i, j)-c(j) \bar{F}(i, j)}{\sigma_{F}(i, j)}\right)^{2}+\sum_{j=5}^{7}\left(\frac{c(j)-\bar{c}(j)}{\sigma(c(j))}\right)^{2},
$$

where $i$ runs over the five dust regions and $j$ runs over the seven bands, $F(i, j)$ is the uncalibrated TopHat or DIRBE flux for region $i$ in band $j, \bar{F}(i, j)$ is the model flux from that region in that band, $c(j)$ is the calibration for that band (a free parameter in every band but the $245 \mathrm{GHz}$ TopHat channel), $\sigma_{F}(i, j)$ is the uncertainty on the uncalibrated flux in that region and band, $\bar{c}(j)$ is the nominal value of the calibration in the three DIRBE bands, and $\sigma(c(j))$ is the uncertainty on that value. The color correction $K(i, j)$ for band $j$, given the model spectrum for region $i$, is the scaling that accounts for the fact that the effective center frequency for a band with finite width will be different depending on the assumed source spectrum. The method for computing the color corrections is explained in Appendix A. The fit is done iteratively, which allows us to apply a color correction at each iteration derived from the source spectrum determined in the previous iteration. This gives a fit with 35 data points and 21 parameters and thus 14 degrees of freedom.

The best fit to the data has $\chi^{2} /$ d.o.f $=36 / 14$, with most of the excess $\chi^{2}$ coming from the TopHat points. The fit residuals are not obviously systematically distributed, implying that the high $\chi^{2} /$ d.o.f may simply be due to an underestimation of the uncertainty, rather than an inappropriate model. We have tested this by performing the calibration fit with a two-component dust model, and find that the $\chi^{2} /$ d.o.f is not improved significantly by the additional parameters, which is consistent with the contention that the poor fit is not due simply to an inadequate model. As we noted in Section 4, there is some evidence of contamination in the maps which is not properly accounted for in the noise covariance matrix, and while we have mitigated this by our choice of regions in the flux analysis and tested for noise misestimation across Epochs (Table 5), there may yet be excess residual contamination which becomes evident when we attempt to combine arbitrary, widely separated regions in the map, as the calibration fit attempts to do. We therefore believe it is justifiable, and conservative, to increase the TopHat uncertainty estimation to account for this discrepancy. We increase the TopHat uncertainty estimation so that the TopHat contribution to the $\chi^{2}$ is comparable to that of the DIRBE points, which is accomplished by doubling the TopHat errors. This improves the $\chi^{2} /$ d.o.f for the one-component dust model calibration fit to $20 / 14$, which has a PTE of 0.13. We conclude from this that a one-component model is an adequate model for the spectra observed, given the best fit gains, if the estimate of the uncertainty of the TopHat fluxes is increased. 
The dust model parameters obtained for each region are given in Table 7 . We note that the assumption of optical thinness of each region is justified a posteriori by these results. Table 7 also gives the square root of the appropriate diagonal element of the covariance matrix for the parameters, which uses the increased TopHat error estimate. This is equivalent to the formal $1 \sigma$ error with marginalization over all other parameters. We stress that the parameters have significant correlations between them. The best fit model for each region, along with its residual, is plotted in Figure 3. 
Table 7. Dust Region Parameters from the Calibration Fit

\begin{tabular}{ccccccc}
\hline \hline Region & $T$ & $\sigma_{T}$ & $\begin{array}{c}\tau\left(\nu_{0}\right) \\
\left(\times 10^{-5}\right)\end{array}$ & $\begin{array}{c}\sigma_{\tau} \\
\left(\times 10^{-5}\right)\end{array}$ & $\alpha$ & $\sigma_{\alpha}$ \\
\hline Dust 1 & 19.4 & 1.0 & .83 & 0.14 & 1.54 & 0.12 \\
Dust 2 & 15.0 & 0.6 & 1.0 & 0.19 & 2.02 & 0.13 \\
Dust 3 & 14.5 & 0.7 & 11. & 2. & 1.98 & 0.16 \\
Dust 4 & 14.8 & 0.6 & 4.4 & 0.8 & 2.06 & 0.15 \\
Dust 5 & 14.7 & 0.6 & 8.0 & 1.5 & 2.00 & 0.15 \\
\hline
\end{tabular}



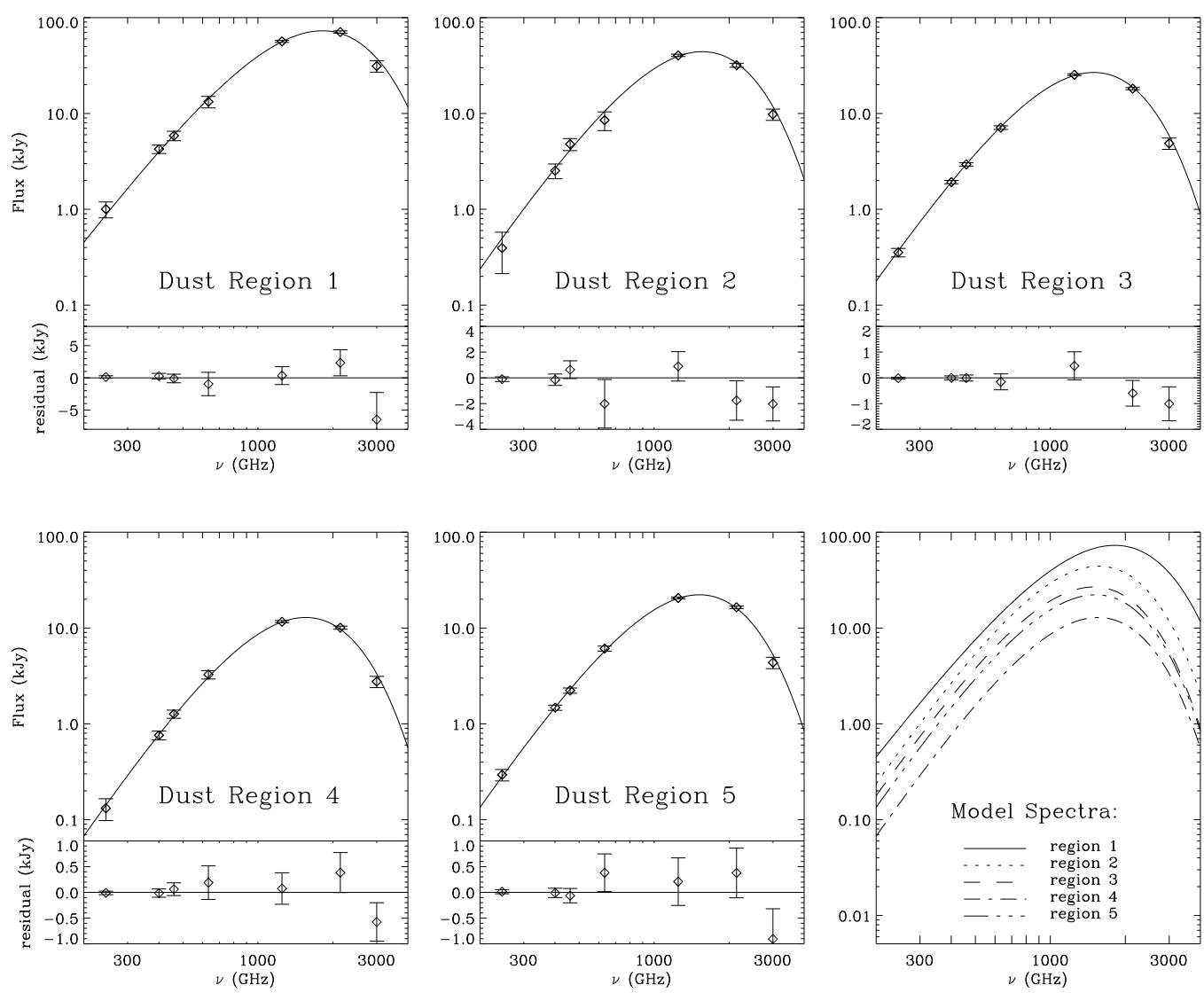

Fig. 3.- Fits to determine the calibration of the TopHat channels at 400, 460, and $630 \mathrm{GHz}$. The best fit model for each of dust regions 1 - 5 is shown, together with the residuals from the fit. All model spectra are plotted together in the lower right panel. 


\section{Calibrated Fluxes}

Using the calibrations obtained from the fit in Section 6 we may then compute the calibrated flux of the LMC excluding 30-Doradus, the SMC, and 30-Doradus alone. The errors in the calibration are uncorrelated with the flux errors for these regions; therefore, the covariance of the calibrated flux is the sum of the covariance of the uncalibrated flux variances and the covariance of the calibration. The calibrated differential fluxes and their errors for these source regions are given in Table 8. The errors quoted are the square root of the diagonal elements of the combined covariance matrix. We then take these spectra and fit them to the model of Equation 7 for each region separately using the full covariance matrix of their errors. The best fit parameters, with errors given by the square root of the diagonal of the covariance matrix, and the $\chi^{2} /$ d.o.f for each of the fits are given in Table 9. The resulting fitted parameters are highly correlated, particularly the emissivity and temperature; the correlation matrices for the fits are given in Table 10. The data, best fit spectra, and fit residuals are shown in Figure 4. We point out that the errors on the parameters as given in Table 9 are appropriate $1 \sigma$ errors under the assumption of Gaussian random errors in the underlying fluxes, and the error for any parameter individually assumes the other parameters are unconstrained. When using all parameters together to describe the flux, it is necessary to consider the correlation as given in Table 10 in propagating the

error; doing so is the only way to capture the full constraint placed on the spectrum by this measurement. 
Table 8. Calibrated fluxes and errors for the LMC, SMC, and 30-Doradus.

\begin{tabular}{cccccccc}
\hline \hline \multirow{2}{*}{ Instrument } & \multirow{2}{*}{$\begin{array}{c}\text { Frequency } \\
(\mathrm{GHz})\end{array}$} & \multicolumn{2}{c}{ LMC } & \multicolumn{2}{c}{$30-$ Dor } & \multicolumn{2}{c}{ SMC } \\
& 245 & 1.63 & 0.17 & 0.27 & 0.03 & 0.32 & 0.08 \\
\multirow{2}{*}{ TopHat } & $245)$ & $\sigma_{F}(\mathrm{kJy})$ & $F(\mathrm{kJy})$ & $\sigma_{F}(\mathrm{kJy})$ & $F(\mathrm{kJy})$ & $\sigma_{F}(\mathrm{kJy})$ \\
& 400 & 7.93 & 0.59 & 1.26 & 0.10 & 0.95 & 0.19 \\
& 460 & 10.57 & 0.89 & 1.85 & 0.16 & 1.62 & 0.29 \\
& 630 & 29.66 & 2.98 & 4.54 & 0.47 & 3.20 & 0.81 \\
\hline \multirow{2}{*}{ DIRBE } & 1250 & 112.3 & 2.4 & 24.99 & 0.57 & 12.07 & 0.54 \\
& 2140 & 177.7 & 3.8 & 46.29 & 1.04 & 20.03 & 0.92 \\
& 3000 & 130.1 & 17.6 & 39.56 & 5.35 & 16.48 & 2.22 \\
\hline
\end{tabular}

Table 9. Results of model fit to the LMC, SMC, and 30-Doradus.

\begin{tabular}{lccrrrrrr}
\hline \hline Region & $\begin{array}{c}T \\
(\mathrm{~K})\end{array}$ & $\begin{array}{c}\sigma_{T} \\
(\mathrm{~K})\end{array}$ & $\begin{array}{c}\tau\left(\nu_{0}\right) \\
\times 10^{-5}\end{array}$ & $\begin{array}{c}\sigma_{\tau} \\
\times 10^{-5}\end{array}$ & $\alpha$ & $\sigma_{\alpha}$ & $\chi^{2} /$ d.o.f & PTE \\
\hline LMC & 25.0 & 1.8 & 0.99 & 0.01 & 1.33 & 0.07 & $5.25 / 4$ & 0.26 \\
SMC & 29.5 & 2.7 & 0.26 & 0.04 & 0.91 & 0.15 & $1.71 / 4$ & 0.79 \\
30-Dor & 26.2 & 2.3 & 2.1 & 0.4 & 1.50 & 0.08 & $1.65 / 4$ & 0.80 \\
\hline
\end{tabular}


Table 10. Correlation matrices for the model fits to the LMC, SMC, and 30-Doradus.

\begin{tabular}{clccc}
\hline \hline Region & Parameter & $T$ & $\tau\left(\nu_{0}\right)$ & $\alpha$ \\
\hline \multirow{3}{*}{ LMC } & $T$ & 1.0000 & -0.9626 & -0.6684 \\
& $\tau\left(\nu_{0}\right)$ & & 1.0000 & 0.4561 \\
& $\alpha$ & & & 1.0000 \\
\hline \multirow{3}{*}{$30-$ Dor } & $T$ & $T$ & $\tau\left(\nu_{0}\right)$ & $\alpha$ \\
& $\tau\left(\nu_{0}\right)$ & 1.0000 & -0.9633 & -0.6584 \\
& $\alpha$ & & 1.0000 & 0.4430 \\
& & & & 1.0000 \\
\hline \multirow{3}{*}{ SMC } & $T$ & $T$ & $\tau\left(\nu_{0}\right)$ & $\alpha$ \\
\hline & $\alpha$ & 1.0000 & -0.8531 & -0.6992 \\
& & & 1.0000 & 0.3046 \\
& & & & 1.0000 \\
\hline
\end{tabular}



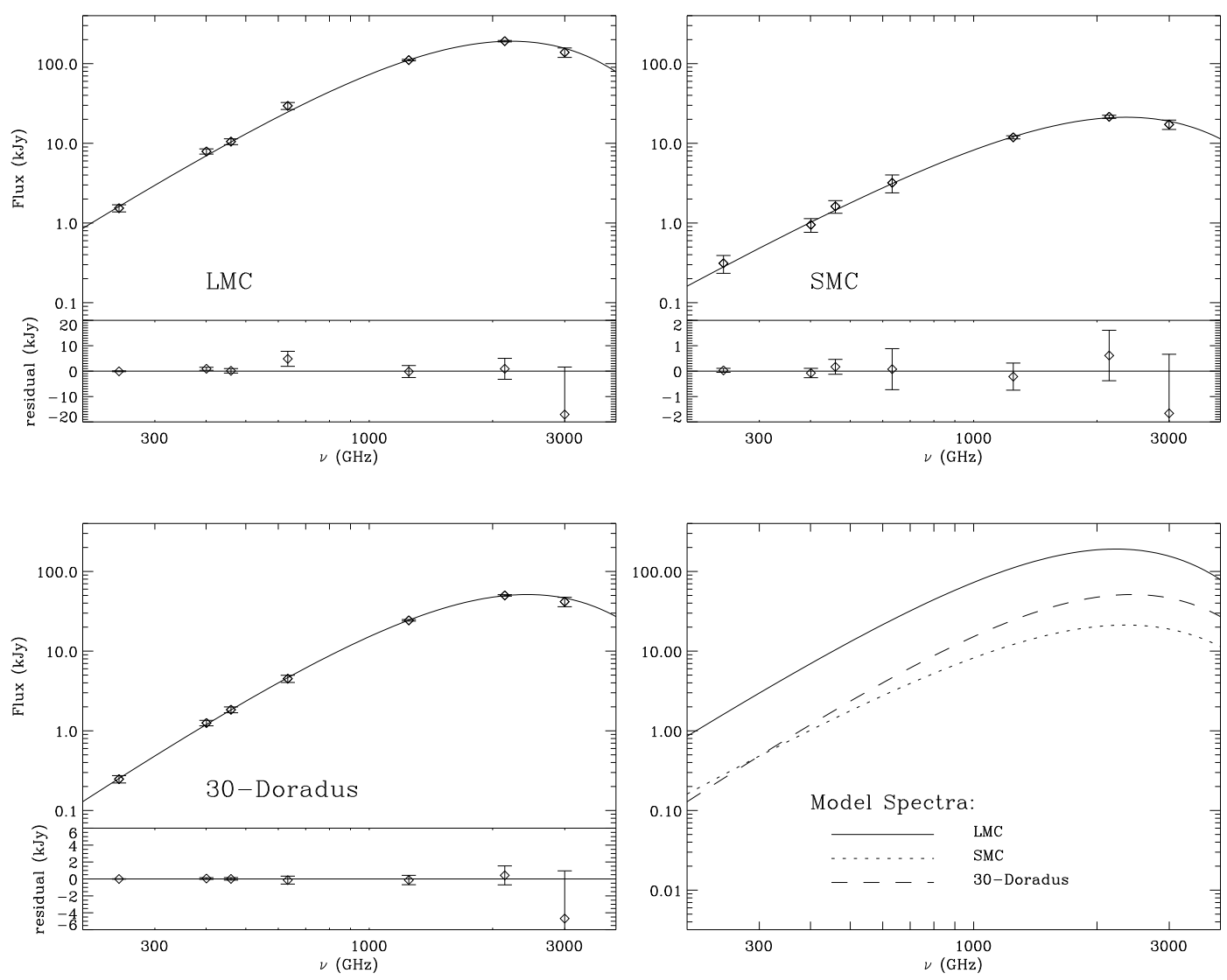

Fig. 4.- Spectrum of calibrated LMC, SMC, and 30-Doradus flux from TopHat and DIRBE. All model spectra are plotted together in the lower right panel. 


\section{Discussion}

We have measured the integrated flux relative to the background of the LMC (minus 30-Doradus), 30-Doradus alone, and the SMC over the frequency range of 245 - $3000 \mathrm{GHz}$. The 245 - $630 \mathrm{GHz}$ measurements (four bands) are new results derived from maps of the Southern Polar Cap made by the TopHat telescope, the first instrument to map this region in these frequency bands with degree-scale angular resolution.

We have applied calibrations to the TopHat $245 \mathrm{GHz}$ data (using the CMB dipole) and the higher-frequency TopHat channels (using an interpolation between the TopHat $245 \mathrm{GHz}$ and DIRBE measurements of five galactic dust regions) and reported integrated fluxes for the LMC, SMC, and 30-Doradus. The only published measurements of the integrated flux of these regions in the millimeter/sub-mm continuum are from Andreani et al. (1990), using timestream data from single scans of a $\sim 1^{\circ}$ beam across the LMC and SMC in two very wide bands $\left(\Delta \nu_{1} \sim 35 \mathrm{GHz}, \Delta \nu_{2} \sim 240 \mathrm{GHz}\right)$ with effective band centers of $\nu_{1}=145 \mathrm{GHz}$ and $\nu_{2}=260 \mathrm{GHz}$. Their reported surface brightnesses are compared to those of this work in Table 11. We are unable to account for the orders-of-magnitude discrepancy in values. We also show the predictions of the FDS99 model in Table 11, and note that the FDS99 predictions are in much closer agreement to our own. As mentioned in Section 1, FDS99 explicitly masks off the Magellanic Clouds in the fit to FIRAS data from which they derive the global parameters of their model. Nevertheless, their prediction for the flux in these regions is useful as an order-of-magnitude guess and is less reliable than their predictions of galactic dust emission only insofar as the optical properties of the dust in the Magellanic Clouds differ from those of the mean high-latitude dust in our galaxy. 
Table 11. Comparison of Previous Surface Brightness Values for the Magellanic Clouds

\begin{tabular}{lccc}
\hline \multicolumn{1}{c}{ Reference } & Center Frequency & \multicolumn{2}{c}{ Surface Brightness } \\
& $(\mathrm{GHz})$ & LMC +30 Dor & SMC \\
\hline Andreani et al. 1990 & 145 & $198 \pm 59$ & $174 \pm 51$ \\
Andreani et al. 1990 & 260 & $1220 \pm 530$ & $905 \pm 440$ \\
FDS99 & 260 & 75 & 25 \\
This work & 245 & $24 \pm 2$ & $12 \pm 3$ \\
\hline
\end{tabular}

Note. - All surface brightnesses given in units of $10^{-18} \mathrm{~W} \mathrm{~cm}^{-2}$ $\mathrm{sr}^{-1} \mu \mathrm{m}^{-1}$ 
We have fit the calibrated TopHat and DIRBE measurements of the LMC (minus 30Doradus), 30-Doradus alone, and the SMC to an emission model which is a blackbody times a power-law emissivity and found that a single temperature and power-law emissivity index fits each of these regions adequately. The regions were fit to a two-component model (with independent temperatures, emissivity power-law indices, and abundances) as well, and the goodness-of-fit did not improve. Stanimirovic et al. (2000) analyzed DIRBE data using their own foreground subtraction process to produce integrated flux from the SMC and found that in a "chi-by-eye" sense (without a formal fit) their fluxes determined from DIRBE band $7,8,9$, and 10 data $(1250 \mathrm{GHz}(240 \mu \mathrm{m})-5000 \mathrm{GHz}(60 \mu \mathrm{m}))$ were not well fit by a single dust component. Similarly, Dunne \& Eales (2001) found that a sample of 32 nearby galaxies observed between $350 \mathrm{GHz}$ and $5000 \mathrm{GHz}$ have spectra more well-described by a two-component model than one with a single component. We also find that if we extend our frequency coverage by adding DIRBE $5000 \mathrm{GHz}$ data (analyzed using the method described in section 4), our spectra are no longer fit well by a single component. But in the frequency range $245 \mathrm{GHz}$ to $3000 \mathrm{GHz}$, we find a single-component fits the emission spectra of the LMC, 30-Doradus, and the SMC just as well as a two-component model.

If we interpret the results of this fit as a physical description of a single dust population in the Magellanic Clouds - rather than a convenient parameterization — we can draw a number of conclusions about the global properties of the dust and the IRF in these regions. Before doing so, however, we note that other physically plausible models could produce the observed spectrum. In fitting FIRAS data to a greybody emission model, Reach et al. (1995) point out that the integrated emission from dust with a broad temperature distribution but a single emissivity power-law index is difficult to distinguish from greybody emission at a single temperature and a shallower power-law emissivity. For example, the spectra from our three regions can be adequately fit by a distribution of emitters with $\alpha=2.0$ (as predicted by the simplest dust models) and a continuous temperature distribution from $T_{d u s t}=T_{c m b}$ up to some maximum temperature $T_{d u s t}=T_{\max }$. For convenience we assume a power-law dust temperature distribution $d N / d T \propto T^{-\beta}$. This gives a model for the flux as

$$
F_{\nu} \propto \int_{T_{c m b}}^{T_{\max }} d T T^{-\beta} B_{\nu}(T)\left(\nu / \nu_{0}\right)^{2.0}
$$

The $\chi^{2} /$ d.o.f and best-fit $T_{\max }$ and $\beta$ from fitting this model to the flux of the LMC, SMC, and 30-Doradus are shown in Table 12. 
Table 12. Results of fit using alternate model of Equation 10.

\begin{tabular}{lccrc}
\hline \hline Region & $T_{\max }$ & $\beta$ & $\chi^{2} /$ d.o.f & PTE \\
\hline LMC & 26.0 & 2.0 & $3.8 / 4$ & .43 \\
SMC & 29.0 & 3.0 & $1.09 / 4$ & .90 \\
30-Dor & 27.5 & 1.4 & $1.92 / 4$ & .75 \\
\hline
\end{tabular}


This alternate interpretation of our results is bolstered somewhat by evidence in previous measurements for significant dust temperature variation within the SMC. Stanimirovic et al. (2000) report SMC dust temperatures of $23 \mathrm{~K}<T_{d}<45 \mathrm{~K}$ based on IRAS 5000/3000 $\mathrm{GHz}$ flux ratios. These values are subject to some scrutiny as absolute temperatures, due both to the offset and calibration issues in the IRAS data and to the fact that temperatures derived from $5000 \mathrm{GHz}$ - to - $3000 \mathrm{GHz}$ flux ratios are probably most sensitive to the nonequilibrium heating and cooling of very small grains. But the observation of variations in this quantity imply a non-uniform IRF in the SMC, which should result in a distribution of temperatures in the larger grains as well.

From our single-component model we derive dust temperatures of (25.0 \pm 1.8$) \mathrm{K}$ for the LMC (minus 30-Doradus), (26.2 \pm 2.3$) \mathrm{K}$ for 30-Doradus alone, and $(29.5 \pm 2.7) \mathrm{K}$ for the SMC. If we instead assume a broad dust temperature distribution, similar values are obtained for the hottest dust in each region (Table 12). The general result that the SMC is hotter than the LMC was seen by Sauvage et al. (1990), who account for this by the lower observed dust-to-gas ratio in the SMC, which would imply more UV photons per dust grain. However, these same authors find that the ratio of $5000 \mathrm{GHz}$ to $3000 \mathrm{GHz}$ emission is higher in 30-Doradus than in the rest of the LMC, while we find the temperatures of these two regions to be within $1 \sigma$ of each other. One would naively expect 30-Doradus to be hotter than the rest of the galaxy because it is an active star forming region with plenty of UV-emitting early-type stars. As in the SMC, the $5000 \mathrm{GHz} / 3000 \mathrm{GHz}$ - derived relative temperature is sampling the transient heating of very small grains, but it implies a more intense IRF in 30-Doradus than in the rest of the LMC, which should be detectable in emission from the larger grains as well. This situation may be better explained by the model of a single emissivity power law and a distribution of grain temperatures. In this model, the maximum dust temperature is similar in 30-Doradus and the rest of the LMC, but the hotter dust makes up a much larger proportion of the total dust in 30-Doradus compared to the rest of the LMC (as evidenced by the best-fit dust temperature power-law indices in the two regions). This makes sense in the context of a simple physical picture in which the hotter dust component is found in the proximity of hot, early-type stars.

Using either of the interpretations of our fit results, we can ask the question "How hot is the dust in the Magellanic Clouds compared to the dust in our galaxy?" FIRAS measured the galactic emission spectrum in all directions between $30 \mathrm{GHz}$ and $3000 \mathrm{GHz}$, and several groups have attempted to derive galactic dust parameters using all or part of the FIRAS coverage. Reach et al. (1995) split the FIRAS coverage into 23 regions in the galactic plane and seven regions above $|b|=10^{\circ}$ and fit these regions separately to a number of different models. They found that in a two-component model with $\alpha=2.0$ for both populations, the hotter component ranged from $18.6 \mathrm{~K} \leq T \leq 24.7 \mathrm{~K}$ in the plane and 
$16.8 \mathrm{~K} \leq T \leq 18.3 \mathrm{~K}$ for the high-latitude regions. FDS99 fit smoothed IRAS and DIRBE data to FIRAS in regions above $|b|=7^{\circ}$, excluding the Magellanic Clouds and HII regions in Orion and Ophiuchus. Their best-fit model was a two-component greybody fit with floating emissivity power-law indices for each component, and the mean temperatures of the two components were $\left\langle T_{1}\right\rangle=9.4 \mathrm{~K}$ and $\left\langle T_{1}\right\rangle=16.2 \mathrm{~K}$. We find that the temperature of the dust in the Magellanic Clouds - or the temperature of the hottest dust component in each of the three observed regions - is on the high side of all of these galactic measurements. The temperature we derive for the SMC is significantly higher than any component from the galactic measurements, while the temperatures we derive for the LMC and 30-Doradus are comparable to the hottest regions seen by Reach et al. (1995) in the plane of our galaxy.

We derive effective emissivity power-law indices of $1.33 \pm 0.07$ for the LMC (minus 30Doradus), $1.50 \pm 0.08$ for 30-Doradus alone, and $0.91 \pm 0.15$ for the SMC. In fitting FIRAS data at high galactic latitudes, Reach et al. (1995) found a similar range of effective powerlaw indices $(0.92<\alpha<1.60)$ for a one-component fit with $\alpha$ as a free parameter. They find slightly better fits with a two-component model and fixed $\alpha=2.0$ but no improvement when they add a slightly broadened uniform temperature distribution. Pollack et al. (1994), in modeling IR dust emission from circumstellar accretion disks at $\sim 100 \mathrm{~K}$ predict an index of $\sim 1.5$ below $500 \mathrm{GHz}$, going over to an index of $\sim 2.6$ at higher frequencies. According to the authors, this is due to the changes in the relative contribution of so-called astronomical silicates - which the authors predict should have an index of 1 - and organic species. In fitting extrapolated and smoothed IRAS and DIRBE data to FIRAS measurements, FDS99 found that a two-component model close to this prediction $\left(\alpha_{1}=1.67, \alpha_{2}=2.7\right.$, with equal power radiated by the two components in the area of $500 \mathrm{GHz}$ ) was the best fit to the high-latitude emission in our galaxy. However, in the three regions we observed we found no evidence for a change in the emissivity power-law index over the observed frequencies (245 $3000 \mathrm{GHz})$.

Finally, we note that if we assume we have sampled a single component of isothermal dust in each region, we can calculate a total mass for that component of dust using the observed optical depth at a particular frequency and a value for the opacity of the observed dust component at that frequency. Of course, published values for low-frequency opacities of likely candidates for astrophysical dust vary widely, and this contribution to the uncertainty in the inferred dust mass dominates the formal uncertainty on the measured optical depth. For example, we can assign an absolute dust opacity to our observed regions by comparing the observed frequency dependence of dust opacity in each region we observed with the measured frequency dependence of various grains in Agladze et al. (1996) and attempting to find a grain species for which their laboratory measurements match our observations. In doing so, we find that at the temperatures we infer for the observed regions, the best 
matches were amorphous $\mathrm{MgO} \cdot 2 \mathrm{SiO}_{2}$ with measured $\alpha \sim 1.1$ at $23 \mathrm{~K}$ for the $\mathrm{SMC}$, and amorphous $2 \mathrm{MgO} \cdot \mathrm{SiO}_{2}$ with measured $\alpha \sim 1.7$ at $25 \mathrm{~K}$ for the LMC and 30-Doradus. The measured absolute opacities of these species at $300 \mathrm{GHz}$ were $3.29 \mathrm{~cm}^{2} \mathrm{~g}^{-1}$ and $1.04 \mathrm{~cm}^{2}$ $\mathrm{g}^{-1}$. Using these values and the $300 \mathrm{GHz}$ optical depth from our best-fit greybody models, we derive integrated dust masses of $(6.2 \pm 1.1) \times 10^{5} M_{\odot}$ for the LMC minus 30-Doradus, $(9.8 \pm 2.1) \times 10^{4} M_{\odot}$ for 30 -Doradus alone, and $(4.0 \pm 0.9) \times 10^{4} M_{\odot}$ for the SMC. (These values

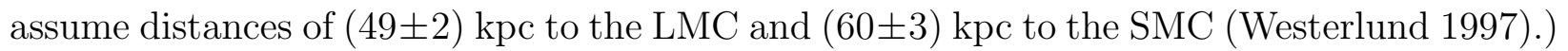
The value thus obtained for the SMC is inconsistent with the value of $(1.8 \pm 0.2) \times 10^{4} M_{\odot}$ obtained in Stanimirovic et al. (2000). However, these authors assumed an absolute dust opacity at $\nu=3000 \mathrm{GHz}$ of $41 \mathrm{~cm}^{2} \mathrm{~g}^{-1}$ (which is close to the DL84 prediction for both graphite and silicate species). And if we apply this value to the $3000 \mathrm{GHz}$ optical depth from our best-fit SMC model, we obtain a value of $(2.6 \pm 0.8) \times 10^{4} M_{\odot}$, which is consistent with the Stanimirovic et al. (2000) result.

However, the greatest uncertainty on this method of calculating dust masses stems from the possibility that the strict physical interpretation of our results is not the correct one. For example, if in fact there is a distribution of temperatures in the SMC with $T_{\max }=30 \mathrm{~K}$ and $d N / d T \propto T^{-3}$, the inferred dust mass will be an order of magnitude higher due to the "hidden" cold dust component. The possibility of hidden cold dust was also recognized in Stanimirovic et al. (2000), whose dust mass calculation was based on temperatures derived from $\nu \geq 3000 \mathrm{GHz}$ data only and was therefore insensitive to dust below $\sim 20 \mathrm{~K}$.

\section{Conclusions}

We have demonstrated that for each of three extragalactic regions (the Large Magellanic Cloud excluding 30-Doradus, 30-Doradus alone, and the Small Magellanic Cloud), the integrated flux in seven frequency bands between $245 \mathrm{GHz}$ and $3000 \mathrm{GHz}$ is well described by a simple, one-component greybody emission model with power-law emissivity. Though it is difficult to obtain robust information about global properties of these regions (such as total dust mass) from these results, the results are intriguing as a potential road map for characterizing the emission in these frequencies across many types of galaxies. If the statistics of emission from extragalactic environments in these frequencies could be accurately described by distributions in a few simple parameters, it would be a boon to groups seeking to probe structure formation by associating the correlation properties of the Cosmic Infrared Background with the distribution of dusty protogalaxies at various redshifts (c.f. Knox et al. (2001)). And knowledge of the "typical" emission in these frequencies from extragalactic environments - and the variation from galaxy to galaxy - is critical for efforts to probe 
the earliest collapsed structures through source counting (c.f. Blain et al. (1999)), because only photometric redshifts will be available for most of the sources.

We are able to put useful constraints on this small set of model parameters for the regions we observe because we have a relative wealth of frequency coverage: seven bands for a threeparameter model. The instruments that give this particular combination of bands (TopHat and $C O B E / D I R B E$ ) have angular resolution that is appropriate for obtaining integrated fluxes from the Magellanic Clouds, but not for extending this technique to a wider sample of galaxies. The IRAS Bright Galaxy Sample is a useful source of information at $\nu \geq 3000$ $\mathrm{GHz}$, and data from the SCUBA instrument has already added points at $350 \mathrm{GHz}$ and 670 $\mathrm{GHz}$ for many of these galaxies (Dunne \& Eales 2001). To obtain constraints of the nature of those we have placed on the Magellanic Cloud flux, however, it will be critical both to augment the sub-mm coverage (which can be achieved with several existing and planned ground-based instruments) and to add points in frequency bands analogous to the DIRBE $2140 \mathrm{GHz}$ and $1250 \mathrm{GHz}$ bands. This area of the spectrum is inaccessible from the ground, so measurements there will have to come from balloon, high-altitude aircraft, or satellite missions. The existing Infrared Space Observatory (ISO) ${ }^{13}$ dataset and anticipated results from the Space Infrared Telescope Facility (SIRTF) ${ }^{14}$ and the Stratospheric Observatory For Infrared Astronomy (SOFIA) ${ }^{15}$ are candidates for filling in this gap. In addition, at lower frequencies, the Wilkinson Microwave Anisotropy Probe (WMAP) ${ }^{16}$ data could be combined with the TopHat and DIRBE data to determine at what frequency other sources of diffuse emission begin to dominate over thermal dust.

We would like to thank the National Scientific Balloon Facility for balloon launch services. We would also like to thank Julian Borrill and acknowledge the use of resources of the National Energy Research Scientific Computing Center. We are also grateful to colleagues at the Danish Space Research Institute and Niels Bohr Institute for their contributions in subsystem development, testing and fabrication and flight support, especially Rene Kristensen and Per Rex Christensen. This research was supported by the NASA Office of Space Sciences and by NSF grant OPP-9619374 and NASA grants NAG5-11443 and NGT5-86/SM.

\footnotetext{
${ }^{13}$ http://www.iso.vilspa.esa.es/

${ }^{14}$ http://sirtf.caltech.edu/

${ }^{15}$ http://sofia.arc.nasa.gov/

${ }^{16}$ http://lambda.gsfc.nasa.gov/product/map/m_products.html
} 


\section{A. Calculation of Color Corrections}

If an experiment has finite bandwidth $\left(t(\nu) \neq \delta\left(\nu-\nu_{c}\right)\right)$, to report a source surface brightness at a single frequency, one must assume a source spectrum. The power detected from that source is assumed to be

$$
P_{\text {in }}=\eta A \Omega \int I_{0}(\nu) t(\nu) d \nu
$$

(Here $\eta$ and $A \Omega$ are the optical efficiency and throughput of the instrument, $I_{0}(\nu)$ is the nominal (assumed) surface brightness of the source, and $t(\nu)$ is the bandpass transmission normalized to 1.0 at its peak.) The effective band center $\nu_{c}$ is usually chosen such that

$$
I_{0}\left(\nu_{c}\right) \simeq \frac{\int I_{0}(\nu) t(\nu) d \nu}{\int t(\nu) d \nu}
$$

The band centers for TopHat are calculated assuming a Rayleigh-Jeans (RJ) source spectrum

$$
\begin{aligned}
I_{0}\left(\nu_{c}\right) & =I_{R J}\left(\nu_{c}\right) \\
& =\tau 2 k T \frac{\nu_{c}^{2}}{c^{2}},
\end{aligned}
$$

where $\tau$ is the optical depth of the source and $k$ is Boltzmann's constant. Since the detected power is assumed to be

$$
P_{i n}=\eta A \Omega \int \tau 2 k T \frac{\nu^{2}}{c^{2}} t(\nu) d \nu
$$

we can write

$$
I_{0}\left(\nu_{c}\right)=\frac{P_{i n}}{\eta A \Omega \int \nu^{2} t(\nu) d \nu} \nu_{c}^{2}
$$

Now if we assume a different source spectrum, for example a greybody with power-law emissivity, the assumed input power is

$$
\begin{aligned}
P_{i n} & =\eta A \Omega \int I_{G B}(\nu) t(\nu) d \nu \\
& =\eta A \Omega \int \tau\left(\nu_{0}\right)\left(\nu / \nu_{0}\right)^{\alpha} B_{\nu}(T) t(\nu) d \nu
\end{aligned}
$$

and the source spectrum inferred from the detected power is

$$
\begin{aligned}
I_{G B}\left(\nu_{c}\right) & =\tau\left(\nu_{0}\right)\left(\nu_{c} / \nu_{0}\right)^{\alpha} B_{\nu_{c}}(T) \\
& =\frac{P_{i n}}{\eta A \Omega \int \nu^{\alpha} B_{\nu}(T) t(\nu) d \nu} \nu_{c}^{\alpha} B_{\nu_{c}}(T) \\
& =\frac{\int \nu^{2} t(\nu) d \nu}{\int \nu^{\alpha} B_{\nu}(T) t(\nu) d \nu} \nu_{c}^{\alpha-2} B_{\nu_{c}}(T) I_{R J}\left(\nu_{c}\right) \\
& \equiv \frac{I_{R J}\left(\nu_{c}\right)}{K} .
\end{aligned}
$$


This defines the color correction $K$ to apply to the reported TopHat flux from a source if the source is assumed to have a greybody spectrum with power-law emissivity.

We make a similar calculation for DIRBE, for which the band centers are computed assuming a spectrum with $\nu I(\nu)$ constant. In this case, the correction is given by

$$
\begin{aligned}
K & =\frac{\nu_{c}^{-1}}{\int \nu^{-1} t(\nu) d \nu}\left[\frac{\tau\left(\nu_{0}\right)\left(\nu_{c} / \nu_{0}\right)^{\alpha} B_{\nu_{c}}(T)}{\int \tau\left(\nu_{0}\right)\left(\nu / \nu_{0}\right)^{\alpha} B_{\nu}(T) t(\nu) d \nu}\right]^{-1} \\
& =\frac{\int \nu^{\alpha} B_{\nu}(T) t(\nu) d \nu}{\int \nu^{-1} t(\nu) d \nu} \nu_{c}^{-(\alpha+1)} B_{\nu_{c}}(T)
\end{aligned}
$$

For an arbitrary experiment with bandpass $t(\nu)$ that reports its surface brightness measurements assuming a spectrum $I_{0}(\nu)$, the surface brightness assuming a different source spectrum $I_{1}(\nu)$ is given by

$$
\begin{aligned}
I_{1}\left(\nu_{c}\right) & =I_{0}\left(\nu_{c}\right) / K \\
& =I_{0}\left(\nu_{c}\right) \frac{I_{1}\left(\nu_{c}\right)}{\int I_{1}(\nu) t(\nu) d \nu}\left[\frac{I_{0}\left(\nu_{c}\right)}{\int I_{0}(\nu) t(\nu) d \nu}\right]^{-1}
\end{aligned}
$$

\section{REFERENCES}

Agladze, N. I., Sievers, A. J., Jones, S. A., Burlitch, J. M., \& Beckwith, S. V. W. 1996, ApJ, 462, 1026

Andreani, P., Ceccarelli, C., dall'Oglio, G., Martinis, L., Piccirillo, L., Pizzo, L., Rossi, L., \& Venturino, C. 1990, ApJ, 348, 467

Blain, A. W., Jameson, A., Smail, I., Longair, M. S., Kneib, J.-P., \& Ivison, R. J. 1999, MNRAS, 309, 715

Borrill, J. 1999, in Proceedings of the 5th European SGI/Cray MPP Workshop, Bologna, Italy, Preprint astro-ph/9911389

Cheng, E. S. 1994, in Lecture Notes in Physics, Vol. 429, Present and Future of the Cosmic Microwave Background, ed. J. L. Sanz et al. (New York: Springer-Verlag), 76

Draine, B. T., \& Lee, H. M. 1984, ApJ, 285, 89

Dunne, L., Eales, S., Edmunds, M., Ivison, R., Alexander, P., \& Clements, D. L. 2000, MNRAS, 315, 115

Dunne, L., \& Eales, S. A. 2001, MNRAS, 327, 697 
Finkbeiner, D. P., Davis, M., \& Schlegel, D. J. 1999, ApJ, 524, 867

Fixsen, D. J., Cheng, E. S., Crawford, T. M., Meyer, S. S., Wilson, G. W., Oh, E. S., \& Sharp, E. H. 2001, RSI, 72, 3112

Fixsen, D. J., \& Mather, J. C. 2002, ApJ, 581, 817

Fixsen, D. J., et al. 1997, ApJ, 490, 482

Gordon, K. D., \& Clayton, G. C. 1998, ApJ, 500, 816

Górski, K. M., Hivon, E., and Wandelt, B. D. 1999. Analysis Issues for Large CMB Data Sets. In Banday, A. J., Sheth, R. S., and da Costa, L., editors, Proceedings of the MPA/ESO Cosmology Conference "Evolution of Large-Scale Structure", PrintPartners Ipskamp, NL, pages 37-42.

Guiderdoni, B., Hivon, E., Bouchet, F. R., \& Maffei, B. 1998, MNRAS, 295, 877

Haiman, Z., \& Knox, L. 2000, ApJ, 530, 124

Hauser, M. G., et al. 1998, ApJ, 508, 25

Hauser, M. G., et al. 1998, COBE Diffuse Infrared Background Experiment (DIRBE) Explanatory Supplement, version 2.3, ed. M. G. Hauser, T. Kelsall, D. Leisawitz, and J. Weiland, COBE Ref. Pub. No. 98-A (Greenbelt, MD: NASA/GSFC), available in electronic form from the NSSDC.

Hildebrand, R. H. 1983, QJRAS, 24, 267

Jaffe, A. H., et al. 2003, Preprint astro-ph/0301077

Kelsall, T., et al. 1998, ApJ, 508, 44

Knox, L., Cooray, A., Eisenstein, D., \& Haiman, Z. 2001, ApJ, 550, 7

Li, A., \& Draine, B. T. 2001, ApJ, 554, 778

Li, A., \& Draine, B. T. 2002, ApJ, 576, 762

Masi, S., et al. 2001, ApJ, 553, L93

Mathis, J. S. 1990, ARA\&A, 28, 37

Mathis, J. S., Rumpl, W., \& Nordsieck, K. H. 1977, ApJ, 217, 425 
Misselt, K. A., Clayton, G. C., \& Gordon, K. D. 1999, ApJ, 515, 128

Oh, E. S., Sharp, E. H., Fixsen, D. J., Cheng, E. S., Inman, C. A., \& Silver, C. 2001, RSI, 72,2735

Pollack, J. B., Hollenbach, D., Beckwith, S., Simonelli, D. P., Roush, T., \& Fong, W. 1994, ApJ, 421, 615

Puget, J. L., et al. 1999, A\&A, 345, 29

Reach, W. T., et al. 1995, ApJ, 451, 188

Rodrigues, C. V., Magalhaes, A. M., Coyne, G. V., \& Piirola, V. 1997, ApJ, 485, 618

Sauvage, M., Vigroux, L., \& Thuan, T. X. 1990, A\&A, 237, 296

Schlegel, D. J., Finkbeiner, D. P., \& Davis, M. 1998, ApJ, 500, 525

Scott, D., et al. 2000, A\&A, 357, L5

Sellgren, K. 1984, ApJ, 277, 623

Silverberg, R. F, et al. 2003, in preparation

Stanimirovic, S., Staveley-Smith, L., van der Hulst, J. M., Bontekoe, T. R., Kester, D. J. M., \& Jones, P. A. 2000, MNRAS, 315, 791

Tielens, A. G. G. M., \& Allamandola, L. J. 1987, in ASSL Vol. 134: Interstellar Processes, 397

van den Bergh, S. 2000, The Galaxies of the Local Group (Cambridge: Cambridge Univ. Press)

Weingartner, J. C., \& Draine, B. T. 2001, ApJ, 548, 296

Westerlund, B. E. 1997, The Magellanic Clouds (Cambridge: Cambridge Univ. Press)

Wheelock, S. L., et al. 1994, IRAS Sky Survey Atlas Explanatory Supplement, JPL Publication 94-11 (Pasadena: JPL) 